\title{
Regulation of intermediary metabolism by protein acetylation
}

\author{
Kun-Liang Guan ${ }^{1,2}$ and Yue Xiong ${ }^{1,3}$ \\ ${ }^{1}$ Molecular and Cell Biology Lab, Institute of Biomedical Sciences, Fudan University, Shanghai \\ 20032, China \\ 2Department of Pharmacology and Moores Cancer Center, University of California San Diego, La \\ Jolla, California 92093 \\ ${ }^{3}$ Department of Biochemistry and Biophysics, Lineberger Comprehensive Cancer Center, \\ University of North Carolina at Chapel Hill, North Carolina 27599
}

\begin{abstract}
Extensive studies during the past four decades have identified important roles for lysine acetylation in the regulation of nuclear transcription. Recent proteomic analyses on protein acetylation uncovered a large number of acetylated proteins in the cytoplasm and mitochondria, including most enzymes involved in intermediate metabolism. Acetylation regulates metabolic enzymes by multiple mechanisms, including via enzymatic activation or inhibition, and by influencing protein stability. Conversely, non-nuclear $\mathrm{NAD}^{+}$-dependent sirtuin deacetylases can regulate cellular and organismal metabolism, possibly through direct deacetylation of metabolic enzymes. Furthermore, acetylation of metabolic enzymes is highly conserved from prokaryotes to eukaryotes. Given the frequent occurrence of metabolic dysregulation in diabetes, obesity, and cancer, enzymes modulating acetylation could provide attractive targets for therapeutic intervention for these diseases.
\end{abstract}

\section{Acetylation in nuclear and cytoplasmic regulation}

Protein lysine acetylation was first reported almost 50 years ago [1-3]. Following the initial discovery of histone acetylation, extensive studies over the last four decades not only have identified the enzymes that catalyze reversible acetylation, the protein lysine acetyltransferases (KATs, formerly termed histone acetyltransferases, HATs) and deacetylases (commonly known as histone deacetylases, or HDACs), but also many nonhistone substrates. Nearly all well characterized acetylation substrates localize in the nucleus, including transcription factors and co-regulators [4,5]. Furthermore, transcription activators and repressors can recruit KATs or HDACs, respectively, to regulate transcription. Collectively, these studies have firmly established the critical functions of acetylation in regulating chromatin structure and gene expression [6-8]. Although cytoplasmic localization of HDACs and KATs has been known for some time, the only well-established cytoplasmic function for acetylation is in regulating microtubule stability by $\alpha$-tubulin acetylation at lysine 40 [9]. Our understanding of the scope of protein acetylation has now changed dramatically following a series of acetylation proteomic studies

\footnotetext{
(C) 2010 Elsevier Ltd. All rights reserved.

Correspondences should be addressed to either K.L.G. (kguan@ucsd.edu) or Y.X. (yxiong@email.unc.edu).
}

Publisher's Disclaimer: This is a PDF file of an unedited manuscript that has been accepted for publication. As a service to our customers we are providing this early version of the manuscript. The manuscript will undergo copyediting, typesetting, and review of the resulting proof before it is published in its final citable form. Please note that during the production process errors may be discovered which could affect the content, and all legal disclaimers that apply to the journal pertain. 
[10-15]: more than 2,000 acetylated proteins have been identified (Box 1), and most are localized in the cytoplasm. Supporting these proteomic studies, genome-wide genetic interaction analyses in budding yeast have linked both KATs and HDACs to many nonnuclear proteins $[16,17]$. These studies have substantially expanded the spectrum of acetylation regulation to most, if not all, major cellular processes. Several excellent reviews have been written on the genetic and cellular function of both protein acetyltransferases and deacetylases in metabolism [18-20]. In this review, we will discuss acetylation-mediated regulation of a specific cellular process, intermediary metabolism, with a focus on how acetylation regulates metabolic enzymes.

\section{Box 1. More than 2,000 proteins are acetylated in mammalian cells}

Owing to the technical difficulty of detection, an overwhelming proportion of the acetylation studies has been focused on histones and nuclear proteins [4,5]. Unlike phosphorylation, which can be readily detected by ${ }^{32} \mathrm{P}$-phosphate labeling, the low specific activity of either ${ }^{3} \mathrm{H}$ or ${ }^{14} \mathrm{C}$ of acetyl group makes it difficult to analyze global cellular protein acetylation and to detect low abundant acetylated proteins. Furthermore, acetylation of $\mathrm{N}$-terminal residues, which is a conserved and widespread modification of most nascent proteins in eukaryotes and catalyzed by a different family of enzymes, $\mathrm{N}-\alpha$ acetyltransferases (NATs) with narrow specificity [21], complicates such an approach because labeling with radioactive acetyl group cannot distinguish whether the modification is on the $\alpha$-amino group of $\mathrm{N}$-terminal residue or on the $\varepsilon$-amino group of lysine side chains of a protein. Although the development of a specific acetyl lysine antibody should have made it possible to globally profile lysine acetylation, its low affinity due to relatively smaller and uncharged acetyl group has made it challenging to efficiently precipitate acetylated proteins. Moreover, acetylated lysine residues can be buried, therefore masking the epitope from the antibody.

Recently, an important improvement in the proteomic identification of acetylated proteins was achieved [10]. This method utilizes the protease digestion of total cellular extracts prior to affinity precipitation using the pan acetyllysine antibody. This digestion probably exposes a greater number of acetylated lysines and has helped significantly to enrich the acetylated peptides. Because the acetylated lysine residue is resistant to trypsin digestion, this method also provides an independent confirmation of mass spectrometry (MS) identification of acetylated peptides. Using this improved purification scheme, combined with increased sensitivity of MS, Kim et al identified 388 lysine acetylation sites corresponding to 195 distinct proteins from mouse liver tissues and HeLa cells [10]. Notably, 277 acetylated peptides are derived from 133 proteins that are located in the mitochondrion, including many intermediary metabolic enzymes. The identification of multiple acetylated metabolic enzymes offered the first peek into the extent of acetylation-mediated regulation of metabolism, gratifyingly meeting the expectation from early studies of mitochondrial localized SIRTs that have long suggested the functions of acetylation in metabolic regulation.

Two subsequent acetylation proteomic studies using similar approaches significantly expanded the acetylome of mammalian cells $[11,15]$. Taking advantage of continuous improvement of MS and adding further steps such as subcellular fractionation to enrich acetylated peptides, these two studies identified an astonishing 1,750 acetylated proteins from a human acute myeloid leukemia cell line [11] and 978 acetylated proteins from human liver tissue [15]. Interestingly, the acetylated proteins identified from human liver tissue overlap more with those identified from mouse liver than to those identified in human lymphocytes, suggesting distinct patterns of acetylation in different tissues that are evolutionarily conserved. Together, these three acetylation studies identified nearly 2,200 acetylated proteins in mammalian cells, making the regulatory scope of acetylation 
comparable to those by other major post-translational modifications such as phosphorylation and ubiquitylation [22]. These acetylated proteins span a wide spectrum of protein classes, ranging from transcription factors, to kinases, ubiquitin ligases, ribosomal proteins and metabolic enzymes, and covering a broad ranges of cellular activities including cell cycle control, DNA damage checkpoints, cytoskeleton organization, endocytosis, and metabolism.

\section{Three SIRTs are localized in mitochondria and linked to metabolic regulation}

Reversible acetylation is controlled by the opposing activities of protein acetyltransferases (KATs) and deacetylases (HDACs and SIRTs), and both are encoded by multigene families with mammalian cells expressing at least 30 acetyltransferases and about 18 deacetylases; all are nuclear encoded. The 18 protein deacetylases are divided into four classes. Class I and II, consisting of 10 family members, are referred to as "classical" HDACs; their enzymatic activity can be inhibited by trichostatin A (TSA). HDAC11 is the only class IV HDAC, and it is insensitive to TSA. Class III protein deacetylases, commonly referred to as SIRTs (or sirtuins, derived from its founding member, budding yeast silent mating type information regulation two protein), includes 7 members in mammalian cells that are structurally unrelated to HDACs, require nicotinamide adenine dinucleotide $\left(\mathrm{NAD}^{+}\right)$as a co-substrate and can be inhibited by nicotinamide (NAM), but not by TSA [23]. Increasing evidence links the functions of multiple SIRTs with metabolic regulation, but much less is known about the function of KATs and HDACs in this mode of regulation.

Of the 11 HDACs, four (HDAC1, 2, 8 and 11) are localized in the nucleus and 7 (HDAC3, $4,5,7,9$ and 10) are either distributed in or shuttle between the nucleus and the cytoplasm [24]. Only one HDAC, HDAC7, has been reported to localize to the mitochondria [25]. Of the 7 mammalian SIRTs, three (SIRT3, 4, and 5) localize in the mitochondria, one (SIRT2) in the cytoplasm, and one (SIRT1) in both the nucleus and the cytoplasm $[4,26]$. The localization of multiple HDACs and SIRTs in the cytoplasm was an early indication of a non-nuclear function of protein acetylation. The mitochondrial localization of multiple SIRTs also suggests a possible role in metabolic regulation as many metabolic reactions occur in mitochondria [27,28]. Genetic studies of various SIRTs in different model organisms, including Saccharomyces cervisiae, Caenorhabditis elegans, Drosophila melanogaster, and mice, have implicated the SIRT genes in lifespan control, caloric restriction and other nutrient responses that are directly linked with metabolic regulation [19,20,29-31]. These studies provide genetic evidence to support a role for SIRTs, and by extension for acetylation, in metabolic regulation.

Do SIRT deacetylases function in metabolic regulation? SIRTs use $\mathrm{NAD}^{+}$as an obligatory substrate in their deacetylation reaction. A high ratio of cellular $\mathrm{NAD}^{+}: \mathrm{NADH}$ indicates a low energy status, raising an intriguing possibility that, like the AMP-activated protein kinase (AMPK) that senses the AMP:ATP ratio and thus cellular energy status, one or multiple mitochondrial or cytoplasmic SIRTs might link extracellular nutrient levels to the acetylation of various metabolic enzymes inside the cell through sensing the intracellular $\mathrm{NAD}^{+}: \mathrm{NADH}$ ratio. Several examples in mammalian cells are consistent with this notion, however further studies are needed to directly support this model. Glucose deprivation impairs differentiation of skeletal myoblasts and is associated with AMPK activation and increased transcription of the $\mathrm{NAD}^{+}$biosynthetic enzyme NAMPT, leading to an increased $\mathrm{NAD}^{+}: \mathrm{NADH}$ ratio and SIRT1 activity. Sirt1+/- myoblasts are more resistant to glucose restriction, supporting the notion that nutrient conditions could affect intracellular activity of 
a SIRT through alterations in NAD+ levels $[32,33]$. Three targets of SIRT1, proliferatoractivated receptor- $\gamma$ coactivator $1 \alpha$ (PGC-1 $\alpha$ ), the forkhead box O1 (FOXO1) and O3 (FOXO3a), were identified, all functioning as transcriptional regulators in the nucleus, leaving the question still open as to whether any cytoplasmic and mitochondrial metabolic enzymes could be directly regulated by SIRT1 which localizes in both the cytoplasm and the nucleus.

In fasted rats, both NAMPT and NAD+ levels increase in liver, leading to elevated SIRT3 activity and increased cell survival [34]. Fasting also increases NAD+ levels and SIRT5 activity in mouse liver, resulting in a decreased acetylation and increased activity of a SIRT5 substrate carbamoyl phosphate synthetase 1 (CPS1) [35], a urea cycle enzyme which catalyzes the initial step of the urea cycle for ammonia detoxification (Fig. 1E). It should be pointed out that in these studies, only NAD+ levels were measured, and a mechanistic basis for SIRTs to sense the ratio of NAD+:NADH instead of being simply dependent on NAD+ for their activity has not been shown. In fact, an in vitro study on SIRT2 found that NADH is an extremely ineffective inhibitor with an average $\mathrm{IC}_{50}$ of an astounding $17 \mathrm{mM}$, leading to the suggestion that physiological NAD+:NADH ratios have little effect on the SIRT2 activity [36].

Analyses of different mitochondrial Sirt genes using mouse knock-out models have been developed over the past few years and have provided genetic support for the function of individual Sirt genes in the regulation of metabolic enzymes. Although developmentally normal, mice with a singular deletion of Sirt3, Sirt4 or Sirt5 exhibit defects in the activity of various metabolic enzymes, including defects in basal ATP levels and fatty-acid oxidation in Sirt3 null mice [37-39], abnormally increased activity of glutamate dehydrogenase (GDH) activity and insulin secretion in Sirt4 null mice [40], and decreased activity of CPS1 and reduced urea cycle function in Sirt5 null mice [35].

A critical piece for the understanding of metabolic regulation by acetylation missing from these genetic and functional studies is the substrates of the cytoplasmic and mitochondrial protein acetyltransferases and deacetylases. This vacuity has now been filled quickly and plentifully by the flurry of recent acetylation proteomic studies [10-12,15], which have identified a large number of acetylated protein as metabolic enzymes. Moreover, emerging data support a role of acetylation in regulating metabolic enzyme functions.

\section{Most metabolic enzymes are acetylated}

Of the nearly 2,200 acetylated proteins identified by acetylation proteomic studies in mammalian cells [10-12,15], metabolic enzymes represent a significant portion of acetylated proteins, due in large part to the use of liver tissues in two of the studies. Nearly all enzymes involved in glycolysis, gluconeogenesis, the tricarboxylic acid (TCA) cycle, fatty acid oxidation, the urea cycle and nitrogen metabolism, and glycogen metabolism are acetylated (Figure 1). Moreover, enzymes involved in oxidative phosphorylation and amino acid metabolism are abundantly acetylated.

The finding that metabolic enzymes are extensively acetylated in tissues or organs such as liver which are engaged in active metabolism was further supported by a smaller and targeted acetylation proteomic study in mouse liver mitochondria [12]. In this study, liver mitochondrial extracts were prepared from mice subjected to a stepwise calorie restriction (CR) protocol over a period of 4 months. Acetylated proteins were affinity purified using a pan anti-acetyllysine antibody, digested with trypsin and analyzed by mass spectrometry (MS). Of the 287 total acetylated proteins identified in this study, at least 165, including many metabolic enzymes, are mitochondrially localized. Semi-quantitative MS analysis identified 72 proteins whose acetylation levels increased during calorie restriction. An 
increase of acetylation in multiple metabolic enzymes during CR is supported by immunoblotting of total liver lysates with the pan anti-acetyllysine antibody. Whether CR increases the overall acetylation of mitochondrial proteins, through activating a protein acetyltransferase(s), inhibiting a deacetylase(s), and/or decreasing the cellular NAD+ concentration, remains be elucidated. The fact that the acetylation levels of the vast majority of metabolic enzymes are increased and very few, if any, are decreased is surprising and suggests that only a limited number of acetyltransferases or deacetylases are involved in the regulation of a large number of metabolic enzymes. Although there is little evidence, the CR-induced acetylation could be caused by a non-enzymatic reaction due to alterations in mitochondrial metabolites, such as acetyl-CoA. Below, we discuss the acetylation-mediated regulation of individual metabolic pathways in greater detail.

\section{Carbon metabolism}

Salmonella enterica acetyl-CoA synthetase (AceS) was the first acetylation-regulated metabolic enzyme to be identified. AceS catalyzes the production of acetyl-CoA from acetate and CoA along with the hydrolysis of ATP to AMP [41]. Acetyl-CoA can enter either the TCA cycle for energy production or the glyoxylate cycle for intermediate biosynthesis and glucose production. The ability of acetyl-CoA to enter the glyoxylate cycle is essential for bacteria to grow on nonfermentable carbon sources, such as acetate or proprionate. S. enterica AceS is acetylated on lysine residue 609, a residue that is essential for AceS catalysis and is highly conserved from prokaryotes to human[41]. Indeed, elegant biochemical experiments showed that acetylation of lysine 609 blocks the formation of the adenylate intermediate, but does not affect the thioester forming activity of AceS. As expected, acetylation strongly inhibits AceS activity both in vitro and in vivo. S. enterica has one SIRT family member, CobB. AceS purified from a $\operatorname{cobB}$ mutant strain is $\sim 100$ fold less active than that purified from wild type cells. Moreover, $c o b B$ mutant cells grow poorly on proprionate or acetate medium. Furthermore, in vitro treatment with recombinant CobB strongly increases the activity of purified AceS. These data have convincingly established that acetylation inhibits AceS activity.

Two independent studies showed that the mammalian AceS is similarly regulated by acetylation on the corresponding lysine residues [42,43]. Mammalian cells have two AceS isoforms, one expressed in the cytoplasm (AceS1) and the other in the mitochondrion (AceS2). Both AceS proteins share strong homology with the bacterial AceS and produce acetyl-CoA. However, the acetyl-CoA produced by AceS1 and AceS2 enters different metabolic pathways: whereas the acetyl-CoA produced by the cytosolic AceS1 provides a critical substrate for biosynthesis such as fatty acid synthesis, the acetyl-CoA generated by the mitochondrial AceS2 is used for cellular energy production by entering the TCA cycle. Biochemical experiments show that purified AceS1 can be acetylated in vitro by a bacterial protein acetyltransferase, PAT. PAT acetylates AceS1 primarily on lysine 661, which is equivalent to lysine 609 in the S. enterica AceS. Similar to the results observed with the bacterial AceS, in vitro acetylation also inactivates mammalian AceS1. Acetylated AceS1 can be efficiently deacetylated by SIRT1, but not SIRT2, and deacetylation results in AceS1 activation. Similar experiments with the mitochondrial AceS2 showed that it is also inactivated by in vitro acetylation with PAT. The mitochondrial SIRT3 has been suggested to deacetylate and activate AceS2 [42,43]. Therefore, reversible acetylation appears to be an ancient mechanism involved in the regulation of AceS. Glyceraldehyde 3-phosphate dehydrogenase (GAPDH), encoded by GapA in S. enterica, catalyzes the reversible reaction between the conversion of glyceraldehyde 3-phosphate and 1,3-bisphosphoglycerate in both glycolysis and gluconeogenesis. Therefore, this enzyme is required for S. enterica to utilize either glucose or acetate as a carbon source. A recent report shows that GAPDH is acetylated, and that its acetylation is strongly increased in cobB mutant Salmonella [14]. 
Interestingly, GAPDH acetylation is higher in cells grown on glucose than those grown on acetate, indicating that GAPDH acetylation is regulated by different carbon sources. PAT mediated GAPDH acetylation increases the glycolytic activity of GAPDH, but inhibits the ability of GAPDH to promote the gluconeogenesis. Conversely, deacetylation by CobB increases GAPDH gluconeogenic activity, but inhibits its glycolytic activity. These observations suggest an exciting model wherein GAPDH acetylation might play a key role in regulating the direction of carbon flux in response to differential nutrient availability. Under glucose-rich conditions, high GAPDH acetylation favors glycolysis; therefore glucose will be used as carbon source for growth. By contrast, under acetate-rich conditions, decreased GAPDH acetylation favors the gluconeogenic direction of the pathway. Therefore, acetate can be used to generate intermediates for biosynthesis and growth. The precise mechanism of this intricate regulation of GAPDH acetylation is not fully understood; however, it might stem from the transcriptional regulation of $\mathrm{Pat}$ and $\mathrm{CobB}$ gene expression in response to different carbon sources. It is worth noting that mammalian GAPDH is also acetylated; it would be interesting to test whether it is similarly regulated and how cellular metabolic conditions might control this mode of regulation.

In addition to its well known function in glycolysis, human GAPDH has also been found in and is essential for the function of the multi-component Oct-1 coactivator (OCA-S) complex, which is essential for $\underline{\mathrm{S}}$ phase-dependent histone H2B transcription [44]. GAPDH binds the $H 2 B$ promoter both in vivo and in vitro and more importantly, the activity of OCA$\mathrm{S}$ is modulated by the cellular NAD+:NADH ratio, suggesting that the involvement of GAPDH in Oct-1 function might link the cellular energy status to cell cycle control. As many as six acetylated lysine residues have now been identified in GAPDH $[11,15]$. More recently, a direct regulation of GAPDH by acetylation was reported [45]; GAPDH associates with and is acetylated by overexpressed PCAF (p300/CBP-associated factor) acetyltransferase, leading to GAPDH nuclear translocation. It will be interesting to determine whether acetylation also affects GAPDH activity during glycolysis, thereby coupling glycolytic rate and energy status to cell proliferation via regulated GAPDH nuclear translocation.

Phosphoenolpyruvate carboxykinase (PEPCK) is a rate limiting regulatory enzyme involved in the switch between glycolysis and gluconeogenesis (Fig. 1A) [46]. It catalyzes the irreversible step of converting oxaloacetate to phosphoenolpyruvate. PECK transcription, and protein stability depend on the cell's need for glucose. A protein acetylation microarray assay in budding yeast revealed that the yeast PEPCK is acetylated and deacetylated by the nucleosome acetyltransferase of $\mathrm{H} 4$ (NuA4) complex and Sir2 deacetylase, respectively [47]. Analysis of human liver samples has identified three acetylation sites in PEPCK [15]. Moreover, PEPCK acetylation in cultured cells is regulated by nutrients in the media. High glucose increases PEPCK acetylation whereas addition of amino acids into glucose-free medium decreases PEPCK acetylation. Unlike GAPDH or AceS, acetylation appears to influence PEPCK1 protein stability [15]. The wild type PEPCK protein is destabilized by high glucose because gluconeogenesis is not needed under conditions of glucose sufficiency. Importantly, substitution of three putative acetylation sites to alanine converts PEPCK to be a stable protein even under high glucose conditions. Conversely, substitution of the acetylated lysine residues by glutamine, which might mimic the effect of acetylation, destabilizes PEPCK. Consistent with a role for acetylation in regulating PEPCK stability, inhibition of deacetylase activity by TSA and nicotinamide destabilizes PEPCK even in glucose-free medium. Collectively, these data establish a critical role of acetylation in controlling PEPCK stability in response to extracellular glucose levels. 


\section{TCA cycle}

The TCA cycle, coupled with oxidative phosphorylation, is the major pathway for aerobic energy production. All eight enzymes in the TCA cycle are acetylated in human liver (Fig. 1B) $[10,11,15]$. In depth analyses of malate dehydrogenase (MDH), which utilizes $\mathrm{NAD}^{+}$as a co-factor to catalyze the reversible oxidation of malate to oxaloacetate, show that as many as four lysine residues in MDH are acetylated. Several lines of evidence indicate that acetylation activates MDH [15]. First, acetylation of MDH is stimulated by increasing glucose concentrations in the culture medium. One might expect that high glucose would promote cell metabolism and thus increase TCA cycle activity. Second, treatment of cells with the deacetylase inhibitors TSA and nicotinamide increases MDH activity; this increase is observed only for wild type MDH but not a mutant MDH in which the four acetylated lysine residues are replaced by arginine. Third, in vitro deacetylation of MDH by purified bacterial CobB deacetylase reduces MDH activity. Furthermore, increasing the concentration of glucose stimulates the activity of wild type MDH, but not the acetylation site mutant. These results indicate that MDH activity is activated by acetylation and that this regulation is involved in the cellular response to glucose.

Acetylation appears to inhibit the activity of another TCA enzyme, succinate dehydrogenase flavoprotein subunit (SDHA), the catalytic subunit of the four-subunit complex that oxidizes succinate to fumarate [48]. SDHA acetylation is elevated, whereas its activity is lower, in Sirt3 knockout mice, thereby identifying SIRT3 as a SDHA deacetylase and ascribing a role for SIRT3 in SDHA activation. Acetylation of isocitrate dehydrogenase 2 (IDH2), which, although it does not directly participate in the TCA cycle, catalyzes $\mathrm{NADP}^{+}$-dependent oxidative decarboxylation of isocitrate to $\alpha$-ketoglutarate in mitochondria, also appears to inhibit its enzymatic activity. SIRT3-mediated deacetylation of purified IDH2 results in increased IDH2 enzymatic activity, supporting the idea that acetylation inhibits IDH2. The fact that acetylation has different effects on the activity of three TCA cycle enzymes, stimulating MDH and inhibiting both SDH and IDH2, indicates the complex nature of this regulation, including the specificity between modifying enzymes, acetyltransferases, deacetylases, and substrates and distinct effects of acetylation on the enzymatic activity.

\section{Lipid metabolism}

A role for acetylation in lipid metabolism can be inferred by the acetylation-induced inhibition of AceS [42,43]. Acetyl-CoA produced by AceS1 can be directly used for fatty acid synthesis in the cytoplasm. More recent findings show that acetylation can directly regulate enzymes involved in fatty acid oxidation. The bifunctional enzyme enoyl CoA hydrotase/3-hydroxylacyl CoA dehydrogenase (EHHADH) catalyzes two sequential steps during fatty acid $\beta$ oxidation, hydration and oxidation (Fig. 1D). Acetylation modifies as many as four different lysine residues in EHHADH and increases its enzymatic activity $[11,12,15]$. Both the acetylation level and enzymatic activity of EHHADH are stimulated by the addition of fatty acids to the culture medium. Quantitative MS analyses demonstrate that a significant fraction of EHHADH in transfected cells can be acetylated. As much as half of ectopically expressed EHHADH is acetylated at Lys346, and treatment of cells with TSA and nicotinamide increases the fraction of Lsy346-acetylated EHHADH to as high as 78\%. These results point to the physiological importance of acetylation in regulating EHHADH activity in response to metabolite fuel availability.

Deletion of the mitochondrial Sirt3 gene in mouse causes a global alteration of mitochondrial protein acetylation, indicating that SIRT3 is a major deacetylase in the mitochondrion [37]. The Sirt3 deficient liver shows decreased fatty acid oxidation with a concomitant accumulation of fatty acid oxidation intermediates and triglycerides under fasting conditions. These phenotypes are likely caused by a defect in the regulation of one or 
several enzymes involved in fatty acid metabolism [39]. Indeed, long-chain acyl CoA dehydrogenase (LCAD) is hyperacetylated on lysine 42 in Sirt3 knockout cells, and in vitro deacetylation of recombinant LCAD by SIRT3 increases its activity. These observations demonstrate the importance of acetylation in negatively regulating LCAD activity through the acetylation of a specific lysine residue. The physiological role of LCAD acetylation is further supported by the fact that Sirt3 knockout mice have a reduced ATP level and cold intolerance [38], findings that are consistent with a defect in fatty acid oxidation. Together, these studies demonstrate an important role of acetylation in regulating mitochondrial fatty acid oxidation and identify SIRT3 as a key deacetylase in this mode of regulation.

\section{Amino acid metabolism}

In addition to serving as building blocks for protein synthesis, amino acids can also be used for energy production and other biosynthetic purposes. Many cancer cells require elevated levels of glutamine [49]. Glutamate dehydrogenase (GDH), which catalyzes the reversible reaction of converting glutamate and $\mathrm{NAD}^{+}$to $\alpha$-ketoglutarate, $\mathrm{NADH}$, and ammonium, is required for both glutamine utilization and glutamate synthesis. Indeed, inhibition of GDH activity has been reported to sensitize glioblastoma cells to glucose deprivation [50]. In liver, GDH is acetylated at as many as 11 lysine residues [10,11,15], and its acetylation also increases during mouse CR [50]. Moreover, gain of function mutations in $G D H$ causes congenital hyperinsulinism in infants, a condition characterized by hypoglycemia and hyperammonemia [51].

Two SIRTs, SIRT3 and SIRT4, physically bind and regulate GDH enzyme activity, providing the first example where an individual metabolic enzyme is regulated by more than one SIRT and thus responds to multiple nutrient conditions. In vitro deacetylation of GDH by SIRT3 activates the enzyme [52], indicating that acetylation has an inhibitory role on GDH. Further support for a role of SIRT3 in regulating GDH acetylation is generated by analyzing Sirt3 knockout mice [37]. MS analysis identified GDH as a prominent acetylated protein in the Sirt3 knockout liver. Moreover, GDH immunoprecipitated from Sirt3 knockout, but not the wild type, liver is highly acetylated. Similarly, the anti-acetyllysine antibody can precipitate GDH from the Sirt3 knockout, but not the wild type, liver tissues. These data strongly support that GDH is acetylated in liver and that SIRT3 is a major GDH deacetylase.

SIRT4 is localized in mitochondria; however, no $\mathrm{NAD}^{+}$-dependent deacetylase activity of SIRT4 has been demonstrated [4]. Instead, SIRT4 has NAD ${ }^{+}$-dependent ADP ribosylase activity, which is also sensitive to inhibition by nicotinamide [40]. SIRT4 can physically interact with GDH and ADP-ribosylate GDH in vitro. Importantly, ADP-ribosylation appears to inhibit GDH activity as seen by the significant decrease of GDH specific activity in Sirt4 deficient cells. Indeed, GDH ADP-ribosylation is altered in Sirt4 knockout cells. These results establish a complex regulation of GDH by two different SIRTs: whereas one, SIRT3, catalyzes deacetylation and activates GDH, the other, SIRT4, ADP-ribosylates and inhibits GDH.

\section{Urea cycle and nitrogen metabolism}

The urea cycle is essential for ammonium detoxification, and mutations within genes encoding urea cycle enzymes are common contributing factors to hyperammonemia, especially during infancy [53]. All eight enzymes in the urea cycle and nitrogen metabolism have been identified as being acetylated ([10-12,15], Fig. 1E), and the effects of acetylation on three enzymes, CPS1, ornithine carbamoyltransferase (OTC), and argininosuccinate lyase (ASL), have been experimentally tested [15,35,54]. CPS1 is the first enzyme of the urea cycle, and as many as 12 lysine residues might be acetylated [10,15]. Affinity purification 
followed by MS analysis has identified CPS1 as a SIRT5 interacting protein; this interaction was confirmed biochemically [35]. CPS1 activity is lower in the Sirt5 knockout liver, and in vitro deacetylation by SIRT5 increases CPS1 activity. Interestingly, CPS1 activity in primary hepatocytes is increased by starvation in a SIRT5-dependent manner; this starvation-induced CPS1 activation is blunted in Sirt5 deficient hepatocytes. Furthermore, starvation or a high protein diet increases CPS1 activity in liver of wild type, but not Sirt5 knockout mice. Under starvation conditions, animals must degrade proteins to generate amino acids as nutrients for survival. Therefore, an elevated urea cycle is expected in animals undergoing starvation. Similarly, animals on a high protein diet should have an elevated urea cycle because the large quantity of amino acids generated will be used for catabolism, thus demanding high CPS1 activity. Therefore, the regulation of CPS1 by acetylation provides another excellent example for how acetylation can modulate metabolism under physiological conditions in response to nutrient availability.

Further illustrating the role of acetylation in the regulation of the urea cycle is the finding that acetylation inhibits the enzymatic activity of both OTC and ASL. OTC acts immediately after CPS1 in the urea cycle. OTC mutations are a relatively common genetic disorder in human and its deficiency causes symptoms ranging from newborn fatal to central nervous system dysfunctions. Three different acetylated lysine residues have been identified $[10,15]$. Acetylation of lysine 88 inactivates OTC enzymatic activity by decreasing the affinity for its substrate, carbamoyl phosphate [54]. Notably, lysine 88 substitutions have been found in human patients with urea cycle defects. Similar to the observations made for CPS1, acetylation of lysine 88 is regulated by glucose availability. High glucose increases OTC acetylation and at the same time decreases OTC1 activity, consistent with an inhibitory role of acetylation in regulating OTC activity. Surprisingly, OTC acetylation is increased by the addition of amino acids in the culture medium. The physiological significance of amino acid-induced OTC acetylation is unclear; indeed, one might expect OTC activation under amino acid-rich conditions.

Activity of ASL in the urea cycle is also regulated by acetylation [15]. Mutations in ASL cause aciduria, the second most common neonatal disorder associated with malfunction of the urea cycle [53]. Acetylation of lysine 288 inhibits ASL activity: high glucose inhibits ASL activity by stimulating acetylation, whereas high concentrations of amino acids activate the enzyme by inhibiting ASL acetylation. The urea cycle is coupled to amino acid metabolism by detoxification of ammonium, and to the TCA cycle because fumarate produced by ASL can be fed into the TCA cycle. A dual regulation of ASL by glucose and amino acids might provide a possible mechanism of pathway coordination. For example, glucose sufficiency would inhibit the utilization of amino acids for energy production. By contrast, under glucose starvation, cells might hydrolyze non-essential proteins/organelles (e.g. by autophagy) to produce amino acids for survival. Increased amino acid catabolism for energy production would demand higher urea cycle activity to remove toxic ammonium. Therefore, cells might utilize acetylation as a mechanism to coordinate multiple metabolic pathways as a way to adapt to alterations in the environment.

\section{Acetylation-mediated metabolic regulation is highly conserved during evolution}

Given the tremendous difference in their nutrient supply and divergence in the regulation of many other cellular activities such as growth and proliferation, it is remarkable that acetylation-mediated regulation of metabolic enzymes is highly conserved from prokaryotes to eukaryotes, both in its extensiveness and mechanistic details. Two acetylation proteomic studies have been conducted with E. coli and Salmonella [13] [14]. Both reports showed that acetylation is highly prevalent in metabolic enzymes. For example, among the 91 acetylated 
proteins identified in E. coli, more than 50\% are metabolic enzymes; by contrast, only two transcription factors were found to be acetylated [13]. This would indicate that more than half of the acetylated proteins are directly involved in metabolism. Although a systematic proteomic acetylation study has not been performed for model eukaryotic organisms such as yeast, C. elegans, and Drosophila, one might anticipate that metabolic enzymes in these organisms are acetylated at a high frequency. Notably, many of the same enzymes are acetylated in both bacteria and human. As exemplified by AceS, inactivation by acetylation is a common regulatory mechanism in both Salmonella and mammals [41-43]. Moreover, analogous lysine residues that are critical for catalytic activity are acetylated across species, and the $\mathrm{NAD}^{+}$-dependent SIRT family deacetylases are responsible for AceS activation. Hence, acetylation appears to be an ancient mechanism for regulating metabolism, and this post-translational modification seems to be widely used in regulating many, if not most, metabolic enzymes, thus supporting a fundamental role of acetylation in metabolic regulation.

\section{Concluding remarks}

In addition to having a major role in regulating gene expression[1-3], recent studies have revealed that acetylation plays a key role in coordinating overall cellular metabolism[10-15]. The extent to which acetylation regulates metabolism is comparable to well-established post-translational modifications such as phosphorylation in the regulation of cell signaling or ubiquitylation in the control of the cell cycle. Similar to regulation by phosphorylation, acetylation appears to have divergent effects on substrates, including inhibition, activation, and regulation of protein stability. These exciting discoveries reveal a new paradigm in metabolic regulation and also raise a number of outstanding questions.

\section{Why are so many enzymes in a given pathway acetylated?}

Most metabolic pathways are not linear; rather, they form a network with many branches from each pathway sharing common intermediates. Therefore, acetylation of multiple enzymes might provide a mechanism of coordinating different pathways. For example, nearly all enzymes involved in glycogen metabolism are acetylated ${ }^{16}$ (Fig. 1C). Glycogen synthesis and glycogen hydrolysis must be coordinated to maintain certain levels of glucose concentration and glycogen storage. Acetylation of these enzymes in glycogen synthesis and hydrolysis could provide a means for such coordination. We propose that acetylation not only regulates the rate of individual pathways but also has a critical role in coordinating the entire metabolic network.

\section{Does the concentration of Acetyl-CoA and NAD+ globally influence the acetylation level of metabolic enzymes?}

Acetyl-CoA is a key intermediate metabolite produced and consumed by many metabolic reactions; as such, its cellular concentration is linked to many metabolic pathways. Thus, any alterations in its concentration might modify and regulate metabolic pathways in response to both extracellular and intracellular metabolic status. Similarly, $\mathrm{NAD}^{+}$is used in most reactions catalyzed by dehydrogenase and its reduced form, NADH, is the major source for energy production by oxidative phosphorylation. Therefore, SIRT-dependent deacetylation might couple cellular metabolic status with intracellular concentrations of a key metabolite, $\mathrm{NAD}^{+}$. One example supporting this notion comes from a study that nuclear histone acetylation in mammalian cells is dependent on ATP-citrate lyase (ACL), the enzyme that converts glucose-derived citrate into acetyl-CoA [55]. 


\section{How do a limited number of acetyltransferases and deacetylases control the acetylation of so many metabolic enzymes?}

Thus far, fewer than 30 protein acetyltransferases and 18 deacetylases have been identified in the human genome. Considering that only three SIRTs and one HDAC (HDAC7) are known to be localized in the mitochondrion, the numbers of enzymes involved in the deacetylation of mitochondrial metabolic enzymes are very small in comparison to the large numbers of metabolic enzymes that are acetylated. The question becomes even more urgent when considering that as many as 2,200 proteins undergo acetylation. By comparison, more than 500 kinases and nearly 150 phosphatases provide a high degree of substrate specificity for phosphorylation. Similarly, as many as 1,000 E3 ubiquitin ligases and 79 deubiquitylating enzymes (DUBs) are expressed or assembled in mammalian cells. Is there a class of yet-to-be-defined factors or mechanism for recruiting specific substrates to an acetyltransferase and/or deacetylase, as is the case for recruiting specific substrates by E3 ubiquitin ligases? Could some metabolic enzymes be acetylated in the cytoplasm before import into mitochondria? Could the high levels of acetyl-CoA directly cause lysine acetylation, which would explain the lack of identification of cytoplasmic and mitochondrial protein acetyltransferases in metabolic regulation?

Understanding these and many other questions emerging from recent studies of acetylation in metabolism will advance our knowledge of metabolic regulation, including an understanding of ways in which metabolic pathways are altered in human disease. In addition to such diseases as cardiovascular diseases, diabetes, obesity and inherited newborn metabolic disorders that have long been linked to dysregulated metabolic enzymes, altered metabolism in tumor cells, that was actually recognized more than 80 years ago by Otto Warburg, is being rediscovered as a key contributor to cancer development. The large number and wide variety of metabolic enzymes, including those from all six main families (oxidases or dehydrogenases, hydrolases, ligases, lyases, isomerases and transferases), offer many potential targets for therapeutic intervention in these metabolic diseases.

\section{Acknowledgments}

We thank Qunying Lei, Guoping Zhao, and Shimin Zhao for their continuous and insightful discussion, and members of Fudan Molecular and Cell Biology Lab for their dedicated works. We are also grateful to three anonymous reviewers for their critical reading and constructive suggestions. This study is supported by NIH grants to K.G. and Y.X.

\section{References}

1. Phillips DM. The presence of acetyl groups of histones. Biochem J 1963;87:258-263. [PubMed: 13943142]

2. Allfrey VG, et al. Acetylation and Methylation of Histones and Their Possible Role in the Regulation of Rna Synthesis. Proc Natl Acad Sci U S A 1964;51:786-794. [PubMed: 14172992]

3. Allfrey VG, et al. Histone acetylation in insect chromosomes. Science 1968;159:314-316. [PubMed: 5634500]

4. Blander G, Guarente L. The Sir2 family of protein deacetylases. Annu Rev Biochem 2004;73:417435. [PubMed: 15189148]

5. Yang XJ, Seto E. Lysine acetylation: codified crosstalk with other posttranslational modifications. Mol Cell 2008;31:449-461. [PubMed: 18722172]

6. Cheung WL, et al. Acetylation and chromosomal functions. Curr Opin Cell Biol 2000;12:326-333. [PubMed: 10801466]

7. Jenuwein T. Re-SET-ting heterochromatin by histone methyltransferases. Trends Cell Biol 2001;11:266-273. [PubMed: 11356363]

8. Yang XJ, Seto E. HATs and HDACs: from structure, function and regulation to novel strategies for therapy and prevention. Oncogene 2007;26:5310-5318. [PubMed: 17694074] 
9. Sullivan KF. Structure and utilization of tubulin isotypes. Annu Rev Cell Biol 1988;4:687-716. [PubMed: 3058169]

10. Kim SC, et al. Substrate and functional diversity of lysine acetylation revealed by a proteomics survey. Mol Cell 2006;23:607-618. [PubMed: 16916647]

11. Choudhary C, et al. Lysine Acetylation Targets Protein Complexes and Co-Regulates Major Cellular Functions. Science 2009;325:834-840. [PubMed: 19608861]

12. Schwer B, et al. Calorie restriction alters mitochondrial protein acetylation. Aging Cell 2009;8:604-606. [PubMed: 19594485]

13. Zhang $\mathbf{J}$, et al. Lysine acetylation is a highly abundant and evolutionarily conserved modification in Escherichia coli. Mol Cell Proteomics 2009;8:215-225. [PubMed: 18723842]

14. Wang Q, et al. Acetylation of metabolic enzymes coordinates carbon source utilization and metabolic flux. Science 2010;327:1004-1007. [PubMed: 20167787]

15. Zhao S, et al. Regulation of cellular metabolism by protein lysine acetylation. Science 2010;327:1000-1004. [PubMed: 20167786]

16. Lin YY, et al. A comprehensive synthetic genetic interaction network governing yeast histone acetylation and deacetylation. Genes Dev 2008;22:2062-2074. [PubMed: 18676811]

17. Mitchell L, et al. Functional dissection of the NuA4 histone acetyltransferase reveals its role as a genetic hub and that Eaf1 is essential for complex integrity. Mol Cell Biol 2008;28:2244-2256. [PubMed: 18212056]

18. Guarente L. Sirtuins as potential targets for metabolic syndrome. Nature 2006;444:868-874. [PubMed: 17167475]

19. Schwer B, Verdin E. Conserved metabolic regulatory functions of sirtuins. Cell Metab 2008;7:104-112. [PubMed: 18249170]

20. Finkel T, et al. Recent progress in the biology and physiology of sirtuins. Nature 2009;460:587591. [PubMed: 19641587]

21. Polevoda B, Sherman F. Nalpha -terminal acetylation of eukaryotic proteins. J Biol Chem 2000;275:36479-36482. [PubMed: 11013267]

22. Choudhary C, Mann M. Decoding signalling networks by mass spectrometry-based proteomics. Nat Rev Mol Cell Biol 2010;11:427-439. [PubMed: 20461098]

23. Smith BC, et al. Mechanisms and molecular probes of sirtuins. Chem Biol 2008;15:1002-1013. [PubMed: 18940661]

24. Riccio A. New endogenous regulators of class I histone deacetylases. Sci Signal 2010;3:pe1. [PubMed: 20051592]

25. Bakin RE, Jung MO. Cytoplasmic sequestration of HDAC7 from mitochondrial and nuclear compartments upon initiation of apoptosis. J Biol Chem 2004;279:51218-51225. [PubMed: 15364908]

26. Michishita E, et al. Evolutionarily conserved and nonconserved cellular localizations and functions of human SIRT proteins. Mol Biol Cell 2005;16:4623-4635. [PubMed: 16079181]

27. Cohen T, Yao TP. AcK-knowledge reversible acetylation. Sci STKE 2004;2004:pe42. [PubMed: 15304664]

28. Huang JY, et al. Mitochondrial sirtuins. Biochim Biophys Acta 2010;1804:1645-1651. [PubMed: 20060508]

29. Haigis MC, Guarente LP. Mammalian sirtuins--emerging roles in physiology, aging, and calorie restriction. Genes Dev 2006;20:2913-2921. [PubMed: 17079682]

30. Anderson RM, Weindruch R. Metabolic reprogramming, caloric restriction and aging. Trends Endocrinol Metab 2010;21:134-141. [PubMed: 20004110]

31. Piper MD, Bartke A. Diet and aging. Cell Metab 2008;8:99-104. [PubMed: 18680711]

32. Fulco M, et al. Glucose restriction inhibits skeletal myoblast differentiation by activating SIRT1 through AMPK-mediated regulation of Nampt. Dev Cell 2008;14:661-673. [PubMed: 18477450]

33. Canto $\mathrm{C}$, et al. AMPK regulates energy expenditure by modulating NAD+ metabolism and SIRT1 activity. Nature 2009;458:1056-1060. [PubMed: 19262508]

34. Yang $\mathrm{H}$, et al. Nutrient-sensitive mitochondrial NAD+ levels dictate cell survival. Cell 2007;130:1095-1107. [PubMed: 17889652] 
35. Nakagawa T, et al. SIRT5 Deacetylates carbamoyl phosphate synthetase 1 and regulates the urea cycle. Cell 2009;137:560-570. [PubMed: 19410549]

36. Schmidt MT, et al. Coenzyme specificity of Sir2 protein deacetylases: implications for physiological regulation. J Biol Chem 2004;279:40122-40129. [PubMed: 15269219]

37. Lombard DB, et al. Mammalian Sir2 homolog SIRT3 regulates global mitochondrial lysine acetylation. Mol Cell Biol 2007;27:8807-8814. [PubMed: 17923681]

38. Ahn BH, et al. A role for the mitochondrial deacetylase Sirt3 in regulating energy homeostasis. Proc Natl Acad Sci U S A 2008;105:14447-14452. [PubMed: 18794531]

39. Hirschey MD, et al. SIRT3 regulates mitochondrial fatty-acid oxidation by reversible enzyme deacetylation. Nature 2010;464:121-125. [PubMed: 20203611]

40. Haigis MC, et al. SIRT4 inhibits glutamate dehydrogenase and opposes the effects of calorie restriction in pancreatic beta cells. Cell 2006;126:941-954. [PubMed: 16959573]

41. Starai VJ, et al. Sir2-dependent activation of acetyl-CoA synthetase by deacetylation of active lysine. Science 2002;298:2390-2392. [PubMed: 12493915]

42. Hallows WC, et al. Sirtuins deacetylate and activate mammalian acetyl-CoA synthetases. Proc Natl Acad Sci U S A 2006;103:10230-10235. [PubMed: 16790548]

43. Schwer B, et al. Reversible lysine acetylation controls the activity of the mitochondrial enzyme acetyl-CoA synthetase 2. Proc Natl Acad Sci U S A 2006;103:10224-10229. [PubMed: 16788062]

44. Zheng L, et al. S phase activation of the histone H2B promoter by OCA-S, a coactivator complex that contains GAPDH as a key component. Cell 2003;114:255-266. [PubMed: 12887926]

45. Ventura M, et al. Nuclear translocation of glyceraldehyde-3-phosphate dehydrogenase is regulated by acetylation. Int J Biochem Cell Biol 2010;42:1672-1680. [PubMed: 20601085]

46. Pilkis SJ, Granner DK. Molecular physiology of the regulation of hepatic gluconeogenesis and glycolysis. Annu Rev Physiol 1992;54:885-909. [PubMed: 1562196]

47. Lin YY, et al. Protein acetylation microarray reveals that NuA4 controls key metabolic target regulating gluconeogenesis. Cell 2009;136:1073-1084. [PubMed: 19303850]

48. Cimen H, et al. Regulation of succinate dehydrogenase activity by SIRT3 in mammalian mitochondria. Biochemistry 2010;49:304-311. [PubMed: 20000467]

49. Deberardinis RJ, et al. Brick by brick: metabolism and tumor cell growth. Curr Opin Genet Dev 2008;18:54-61. [PubMed: 18387799]

50. Yang C, et al. Glioblastoma cells require glutamate dehydrogenase to survive impairments of glucose metabolism or Akt signaling. Cancer Res 2009;69:7986-7993. [PubMed: 19826036]

51. Stanley CA, et al. Hyperinsulinism and hyperammonemia in infants with regulatory mutations of the glutamate dehydrogenase gene. N Engl J Med 1998;338:1352-1357. [PubMed: 9571255]

52. Schlicker C, et al. Substrates and regulation mechanisms for the human mitochondrial sirtuins Sirt3 and Sirt5. J Mol Biol 2008;382:790-801. [PubMed: 18680753]

53. Trevisson E, et al. Argininosuccinate lyase deficiency: mutational spectrum in Italian patients and identification of a novel ASL pseudogene. Hum Mutat 2007;28:694-702. [PubMed: 17326097]

54. $\mathrm{Yu} \mathrm{W}$, et al. Lysine 88 acetylation negatively regulates ornithine carbamoyltransferase activity in response to nutrient signals. J Biol Chem 2009;284:13669-13675. [PubMed: 19318352]

55. Wellen KE, et al. ATP-citrate lyase links cellular metabolism to histone acetylation. Science 2009;324:1076-1080. [PubMed: 19461003]

56. Allis CD, et al. New nomenclature for chromatin-modifying enzymes. Cell 2007;131:633-636. [PubMed: 18022353]

57. Rine J, et al. A suppressor of mating-type locus mutations in Saccharomyces cerevisiae: evidence for and identification of cryptic mating-type loci. Genetics 1979;93:877-901. [PubMed: 397913]

58. Shore D, et al. Characterization of two genes required for the position-effect control of yeast mating-type genes. EMBO J 1984;3:2817-2823. [PubMed: 6098447]

59. Smith JS, et al. A phylogenetically conserved NAD+-dependent protein deacetylase activity in the Sir2 protein family. Proc Natl Acad Sci U S A 2000;97:6658-6663. [PubMed: 10841563]

60. Landry J, et al. The silencing protein SIR2 and its homologs are NAD-dependent protein deacetylases. Proc Natl Acad Sci U S A 2000;97:5807-5811. [PubMed: 10811920] 
61. Imai S, et al. Transcriptional silencing and longevity protein Sir2 is an NAD-dependent histone deacetylase. Nature 2000;403:795-800. [PubMed: 10693811]

62. Ahuja N, et al. Regulation of insulin secretion by SIRT4, a mitochondrial ADP-ribosyltransferase. J Biol Chem 2007;282:33583-33592. [PubMed: 17715127]

63. Liszt G, et al. Mouse Sir2 homolog SIRT6 is a nuclear ADP-ribosyltransferase. J Biol Chem 2005;280:21313-21320. [PubMed: 15795229]

64. Greiss S, Gartner A. Sirtuin/Sir2 phylogeny, evolutionary considerations and structural conservation. Mol Cells 2009;28:407-415. [PubMed: 19936627]

65 . Du J, et al. Investigating the ADP-ribosyltransferase activity of sirtuins with NAD analogues and 32P-NAD. Biochemistry 2009;48:2878-2890. [PubMed: 19220062]

\section{Glossary Box}

\section{Calorie restriction (CR)}

\section{Histone acetyltransferases (HATs)}

\section{Intermediary metabolism}

\section{Protein lysine deacetylases}

\section{Sirtuins (SIRTs)}

a dietary regimen that restricts caloric intake by experimental organisms, in contrast to control organisms that are provided with unrestricted food. CR has a long history of research in a wide range of model organisms, including yeast, C. elegans, Drosophila, and mammals, and consistently has been found to increase both the median and maximum lifespan. Although the mechanism underlying extended life span by CR remains incompletely understood, altered metabolic activity appears to be a major contributing factor, including the alteration in acetylation of metabolic enzymes [12].

a group of enzymes that transfer an acetyl group from acetyl-CoA to form $\varepsilon-\mathrm{N}$-acetyl lysine. Although first discovered as enzymes that acetylate conserved lysine residues on histones and thus so named, recent recommendations suggest renaming them as lysineacetyltransferases (KATs) to reflect their broad function in regulating a great number of non-histone proteins [56], including many metabolic enzymes discussed herein.

often referred to simply as metabolism, includes all steps between extracellular foodstuffs or nutrients and cellular components. These steps are catalyzed by enzymes within the cell and provide energy, reducing power and building blocks for maintaining cellular homeostasis or supporting cell growth.

also known as histone deacetylases, HDACs. These comprise a group of hydrolases that remove acetyl groups from the $\varepsilon$-amine of lysine side chains. Although histone proteins were the first identified HDAC substrates, it is now clear that the HDAC substrate spectrum is much broader. Although HDACs have been referred to in some literature as KDACs to more precisely describe their function toward numerous non-histone proteins, recent recommendations suggest against the renaming to avoid confusion owing to the long history of its reference in the literature [56].

SIRT proteins were named after its founding member Sirtuin, which was derived from silent information regulator twwo (Sir2) proteins, originally identified and then isolated in a screen for mutations that suppresses the mating and sporulation defects in budding yeast[57,58]. They are categorized as class III protein lysine deacetylases that possess $\mathrm{NAD}^{+}$-dependent deacetylase 
[59-61] except for SIRT4, which does not exhibit deacetylase activity and instead possesses $\mathrm{NAD}^{+}$-dependent ADP ribosylase activity $[40,62]$, and SIRT6 which exhibits both $\mathrm{NAD}^{+}$-dependent deacetylase and ADP ribosylase activities [63]. SIRTs are structurally distinct from other classes of HDACs; they represent an evolutionarily conserved multigene family, including two genes in yeast, C.elegans and Arabidopsis thaliana, five in Drosophila, sea urchin and zebrafish, seven in Xenopus laevis, chicken and mammals [64]. The rate of SIRT-mediated ADP-ribosylation, however, seems to be very low [65], which, when considered together with the fact that most eukaryotes express poly-ADPribose polymerases (PARPs) that can catalyze ribosylation more efficiently, raises the question of its physiological relevance and whether it represents a side reaction of deacetylation. 


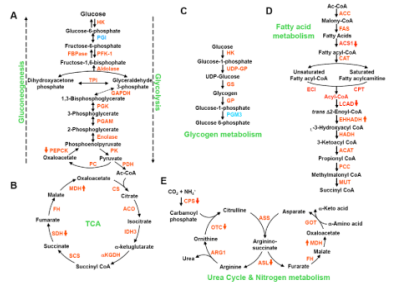

Figure 1. Acetylation regulation of metabolic enzymes

Most intermediate metabolic enzymes are acetylated. The five major pathways that include enzymes whose regulation by acetylation has been characterized-gluconeogenesis (a), glycolysis (b), glycogen metabolism (C), fatty acid metabolism (d) and the urea cycle and nitrogen metabolism (e)--are illustrated. Substrates and products are colored in black.

Metabolic enzymes are colored in red if their acetylations have been identified or blue if not. The red arrows, facing up or down, next to the enzymes indicate a stimulatory or inhibitory effect, respectively, on the enzyme activity by acetylation as determined by in vitro biochemical experiments 